\title{
PLCE1 regulates the migration, proliferation, and differentiation of podocytes
}

\author{
Seyoung Yu', Won-ll Choi $^{2}$, Yo Jun Choi ${ }^{1}$, Hye-Youn Kim', Friedhelm Hildebrandt ${ }^{2}$ and Heon Yung Gee ${ }^{1}$
}

\begin{abstract}
PLCE1 encodes phospholipase C epsilon, and its mutations cause recessive nephrotic syndrome. However, the mechanisms by which PLCE1 mutations result in defects associated with glomerular function are not clear. To address this, we investigated the function of PLCE1 in podocytes called glomerular epithelial cells, where the pathogenesis of nephrotic syndrome converges. PLCE1 colocalized with Rho GTPases in glomeruli. Further, it interacted with Rho GTPases through the pleckstrin homology domain and Ras GTP-binding domains 1/2. Knockdown or knockout of PLCE1 in podocytes resulted in decreased levels of GTP-bound Rac1 and Cdc42, but not those of RhoA, and caused a reduction in cell migration. PLCE1 interacted with NCK2 but not with NCK1. Similar to the PLCE1 knockout, NCK2 knockout resulted in decreased podocyte migration. Knockout of PLCE1 reduced the EGF-induced activation of ERK and cell proliferation in podocytes, whereas knockout of NCK2 did not affect proliferation. Further, the knockout of PLCE1 also resulted in decreased expression of podocyte markers, including NEPH1, NPHS1, WT1, and SYNPO, upon differentiation, but the knockout of NCK2 did not affect the expression of these markers. Therefore, our findings demonstrate that PLCE1 regulates Rho GTPase activity and cell migration through interacting with NCK2 and that PLCE1 also plays a role in the proliferation and differentiation of podocytes, regardless of the presence of NCK2.
\end{abstract}

\section{Introduction}

Filtration is an important function of the kidneys, and the human kidney contains one million glomeruli (filtering units). Glomeruli consist of three cell types: podocytes, endothelial cells, and mesangial cells ${ }^{1}$. Podocytes are neuron-like terminally differentiated epithelial cells that contain three segments: the cell body, major processes, and foot processes. The foot processes are in direct contact with the urinary side of the glomerular basement membrane and interdigitate with the foot processes from neighboring cells through specialized intercellular junctions (the 'slit diaphragm'), thereby forming sieve-like structures that prevent the loss of plasma proteins into the urine $^{1}$. This structure serves to create a maximal filtration

\footnotetext{
Correspondence: Heon Yung Gee (hygee@yuhs.ac)

'Department of Pharmacology, Brain Korea 21 PLUS Project for Medical Sciences, Yonsei University College of Medicine, Seoul 03722, Korea

${ }^{2}$ Department of Medicine, Division of Nephrology, Boston Children's Hospital, Harvard Medical School, Boston, MA 02115, USA

These authors contributed equally: Seyoung Yu, Won-II Choi
}

space between cells while simultaneously supporting and maintaining the glomerular basement membrane ${ }^{2}$.

Defects in the glomerular filter result in nephrotic syndrome, a kidney disease characterized by albumin loss (proteinuria). Nephrotic syndrome is classified as either steroid-sensitive or steroid-resistant, depending on the response to steroids, which are commonly used as firstline therapy ${ }^{3}$. A significant proportion of steroid-resistant nephrotic syndromes (SRNSs) have a genetic background and result from a single-gene defect. The genetic heterogeneity of SRNS can be demonstrated by the fact that mutations in approximately 50 genes have been identified to be capable of causing SRNS ${ }^{4,5}$. Research on the function of genes linked to SRNS has revealed that podocytes are critical sites for the pathogenesis of SRNS ${ }^{6,7}$. SRNS is accompanied by the rearrangement of the actin cytoskeleton and simplification of interdigitating cellular processes of podocytes, called foot process effacement, which is a classic feature of proteinuric kidney diseases, including SRNS ${ }^{7}$. 
Mutations in PLCE1, the gene encoding phospholipase $C$ epsilon 1, have been described in patients with earlyonset nephrotic syndrome ${ }^{8}$. In individuals exhibiting PLCE1 mutations, kidney histology shows diffuse mesangial sclerosis (DMS) in most cases and focal segmental glomerulosclerosis (FSGS) in others ${ }^{9}$. Members of the phosphoinositide-specific phospholipase $\mathrm{C}$ family catalyze the hydrolysis of membrane phospholipids to generate the second messenger molecules inositol 1,4,5trisphosphate $\left(\mathrm{IP}_{3}\right)$ and diacylglycerol (DAG), which initiate intracellular cell growth and differentiation pathways ${ }^{10}$. PLCE1 is activated by $G$ proteins, such as $G_{\alpha 12 / 13}$, and small GTPases, such as Ras and RhoA ${ }^{11,12}$. DAG is an agonist of TRPC6, whose gain-of-function mutations cause late-onset nephrotic syndrome ${ }^{13,14}$. Therefore, it was shown that loss of PLCE1 can lead to disturbed DAGmediated TRPC6 activation. However, podocytes isolated from $\mathrm{Plce}^{-1-}$ mice were indistinguishable from control podocytes with respect to TRPC6 activation, indicating a redundancy in the PLC function in podocytes in this particular context ${ }^{15}$.

The role of PLCE1 in podocytes and the pathogenesis of nephrotic syndrome due to the loss of PLCE1 are not well understood. To address this, PLCE1-deficient podocytes were examined, and it was found that the migration, proliferation, and maturation of podocytes were negatively affected by the loss of PLCE1, thereby revealing a link between PLCE1 mutations and podocytopathy.

\section{Materials and methods}

\section{Plasmids, cell culture, and transfection}

The human PLCE1 clone has been described previously ${ }^{8}$. The fragments of PLCE1 were subcloned into pEGFP-C1 (Clontech, Mountain View, CA, USA). NCK1 and NCK2 clones were purchased from the Harvard PlasmID Database. Immortalized human podocytes were provided by Moin Saleem (University of Bristol, Bristol, UK) and maintained in RPMI plus GlutaMAX-I (Gibco) supplemented with $10 \% \mathrm{FBS}$, penicillin-streptomycin $(50 \mathrm{IU} / \mathrm{ml}$ and $50 \mu \mathrm{g} / \mathrm{ml}$, respectively), and insulin-transferrinselenium-X. Plasmids and siRNA were transfected into podocytes cultured at a permissive temperature of $33^{\circ} \mathrm{C}$ using Lipofectamine 2000 (Invitrogen, Carlsbad, CA, USA). Podocytes were cultured for differentiation at $37^{\circ} \mathrm{C}$ for 14 days. The PLCE1-specific and control scrambled siRNAs were purchased from Qiagen. HEK293 or Lenti-X $293 \mathrm{~T}$ cells were propagated in DMEM supplemented with $10 \% \mathrm{FBS}$ and penicillin-streptomycin $(50 \mathrm{IU} / \mathrm{ml}$ and $50 \mu \mathrm{g} / \mathrm{ml}$, respectively). sgRNAs targeting human NCK2 (sgRNA1, 5'-GCTATGTACCGTCCAACTACG-3'; sgRNA 2, 5'-GTGGACATCAAGAAGAACGAG-3'; and sgRNA3, 5'-GTACCGTCCAACTACGTGGAG-3') and human PLC E1 (sgRNA1, 5'-GCCAGCCTCCGAGACAGCCCA-3'; sgR NA2, 5'-GCCCATGGAAGGATAAGCGT-3'; and sgRNA
3, 5'-GACTTCTTGCTCCCAAAGACG-3') were cloned into BsmBI-digested lentiCRISPR v2 (Addgene \#52961, Watertown, MA, USA). To establish PLCE1 or NCK2 knockout cell lines, lentiCRISPR v2, pMD2.G, and psPAX2 were transfected into Lenti-X 293T cells (Clontech). The supernatant containing lentivirus was collected $48 \mathrm{~h}$ after transfection and passed through a $0.2 \mu \mathrm{m}$ filter. Cultured podocytes were transduced with lentivirus containing lentiCRISPR clones, selected, and maintained in the presence of $4 \mu \mathrm{g} / \mathrm{ml}$ puromycin.

\section{Immunoblotting, GST pulldown, and immunoprecipitation}

These experiments were performed as described previously ${ }^{16}$. GST-Rac1 (Addgene, \#12200) and GST-RhoA (Addgene, \#12202) were purified from the BL21 (DE3) E. coli strain, and GST-PAK1 CRIB and GST-rhotekin RBD domains were purchased from Cytoskeleton, Inc. AntiFLAG, anti-Myc, anti-p-ERK, anti-p-JNK, anti-ERK (Cell Signaling Technology, Danvers, MA, USA), anti-Rac1, anti-Cdc42 (BD Transduction Laboratories), anti-RhoA (Santa Cruz Biotechnology, Dallas, TX, USA), and antiGFP (Invitrogen) were purchased from the indicated commercial sources. The anti-PLCE1 antibody has been previously described by Hinkes et $\mathrm{al}^{8}$. Coimmunoprecipitation was performed using EZview Red anti-FLAG M2 or Anti-c-Myc Affinity Gels (Sigma-Aldrich, St.Louis, MO, USA). The intensities of immunoblots were analyzed using ImageJ.

\section{Immunofluorescence}

The rat kidney tissue was fixed in $4 \%$ paraformaldehyde overnight at $4{ }^{\circ} \mathrm{C}$, embedded in paraffin blocks, and cut into $5 \mu \mathrm{m}$-thick sections. The sections were then mounted on slides, deparaffinized, and rehydrated through a graded series of ethanol concentrations. After rehydration, antigen retrieval was performed by boiling the sections for 30 min using a Retrieve-All Antigen unmasking system 1 (pH 8; BioLegend, San Diego, CA, USA). Sections were permeabilized with $1 \%$ sodium dodecyl sulfate for $10 \mathrm{~min}$ at room temperature, blocked with normal donkey serum (1\% BSA, $10 \%$ donkey serum in PBS) for $1 \mathrm{~h}$, and incubated overnight with primary antibodies. The next day, sections were washed with PBS and incubated with secondary antibodies, Alexa 488 and Alexa 594 (Invitrogen); the sections were then washed with PBS, stained with DAPI and mounted on coverslips. Fluorescence images were obtained using an LSM 780 confocal microscope (Carl Zeiss).

\section{Wound-healing assay}

Cells were seeded in 96-well image lock plates (Essen Bioscience, Ann Arbor, MI, USA). Podocytes were examined for confluency as a monolayer via light microscopy before the initiation of the wound-healing 
assay. Scratches were made using a 96-pin tool (Woundmaker), according to the protocol provided by the manufacturer.

\section{Podocyte migration and proliferation assays}

Real-time migration or proliferation assays were performed using the xCELLigence system (ACEA Biosciences, Inc, San Diego, CA, USA), according to the manufacturer's instructions. Briefly, $2 \times 10^{4}$ cells were seeded in an E-Plate for the proliferation assay and were treated with $100 \mathrm{ng} / \mathrm{ml}$ epidermal growth factor. For the migration assay, $4 \times 10^{4}$ cells were seeded in serum-free media in the upper chamber of a CIM-Plate 16, while the lower chambers were filled with 10\% FBS for chemoattraction or with serum-free media. The data were analyzed using RTCA software. The results are presented as the time-versus-cell index.

\section{Quantitative RT-PCR}

Total RNA was extracted from podocytes and reversetranscribed using the iScriptTM Select cDNA Synthesis Kit (Bio-Rad, Hercules, CA), according to the manufacturer's instructions. Target amplification was performed in 96-well plates using the StepOnePlus ${ }^{\mathrm{TM}}$ Real-Time PCR System and SYBR Green PCR Master Mix (Thermo Fisher Scientific, San Jose, CA, USA), according to the manufacturer's instructions. The primers used in the RT-PCR analysis were GAPDH (5'-CATCAGCAATGCCTCCTG-3', 5'-AGTCCT TCCACGATACCAAAG-3'), NEPH1 (5'-GCCATCTACTC GTCGTTTAAG-3', 5'-GCACGGTAGTCAGCATAGAG3'), NPHS1 (5'-CCTTCTGCAAGTGTCATCC-3', 5'-GTT AGCGGACACGGACAC-3'), SYNPO (5'-AAGTCACATC CAGCTCCTTC-3', 5'-CTTCTCCGTGAGGCTAGTG-3'), and WT1 (5'-CCCTACAGCAGTGACAATTTATAC-3', 5'-TGCCCTTCTGTCCATTTC-3'). All samples were run in triplicate. The relative RNA expression levels were calculated via a comparative threshold cycle $(\mathrm{Ct})$ method using GAPDH as the control: $\Delta \mathrm{Ct}=\mathrm{Ct}(\mathrm{GAPDH})-\mathrm{Ct}$ (target gene). The fold change in the gene expression, which was normalized to GAPDH and relative to the control sample, was calculated as $2^{-\Delta \Delta \mathrm{Ct}}$.

\section{Statistical analysis}

The results are presented as the means \pm SEM or SD for the indicated number of experiments. Statistical analysis of continuous data was performed with a 2-tailed Student's $t$-test or a Mann-Whitney $U$ test, as appropriate. $P<0.05$ was considered statistically significant.

\section{Results}

PLCE1 interacts with RhoA and Rac1 in podocytes

PLCE1 is activated by the direct binding of small GTPases, including Ras and RhoA ${ }^{11,12}$. As Rac1, Cdc42, and RhoA are implicated in the pathogenesis of nephrotic syndrome in mice and humans ${ }^{16-20}$, we examined whether these Rho GTPases interact with PLCE1 in cultured podocytes. Coimmunoprecipitation in the background of PLCE1 overexpression revealed that PLCE1 interacts with Rac1 (Fig. 1a) and RhoA (Fig. 1b) but not with Cdc42 (Fig. 1c). The interaction of PLCE1 with Rac1 and RhoA was further confirmed by the pulldown assay (Fig. 1d, e).

The expression of PLCE1 in the glomeruli was examined. PLCE1 has been reported to be expressed in developing and mature podocytes ${ }^{8}$, and we also confirmed that PLCE1 is enriched in podocyte cell bodies and processes by staining for the podocytic markers WT1 and SYNAPTOPODIN using immunofluorescence (Fig. 1f, g). Immunostaining of PLCE1 with Rac1 showed the colocalization of both proteins in cell bodies and the processes of podocytes (Fig. 1h), whereas PLCE1 colocalized with RhoA mostly in the cell bodies (Fig. 1i).

To identify the domain of PLCE1 that interacts with Rac1 and RhoA, coimmunoprecipitation was performed in cultured podocytes (Fig. 2). PLCE1 contains a RasGEF (a.k.a., CDC25, guanine nucleotide exchange factor for Ras-like small GTPases) domain, a pleckstrin homology $(\mathrm{PH})$ domain, two regions with high sequence conservation among all PLCs (X and Y), a C2 domain, and two Ras-binding (RA) domains (Fig. 2a) ${ }^{12}$. Among these, both Rac1 and RhoA bind to PLCE1 through the PH and RA1/ 2 domains (Fig. 2b, c).

\section{Loss of PLCE1 resulted in decreased active Rac1 and Cdc42 and decreased podocyte migration}

As PLCE1 interacts with Rac1 and RhoA in podocytes, the effect of PLCE1 on the active states of Rho GTPases was examined. PLCE1 was knocked down using siRNAs, and pulldown assays were performed using purified PAK1 and Rhotekin. Knockdown of PLCE1 in cultured podocytes led to a decrease in active GTP-bound Rac1 (43\%) and Cdc42 (40\%) (Fig. 3a-c) but had no effect on the levels of active RhoA in the cultured podocytes (Fig. 3d, e).

As Rho GTPases regulate cell migration ${ }^{21}$ and PLCE1 knockdown alters the active status of Rac1 and Cdc42, podocyte migration was examined using two different approaches. In the wound-healing assay, podocytes with siRNA-mediated PLCE1 knockdown exhibited delayed wound closure compared to the control podocytes (Fig. $4 \mathrm{a}, \mathrm{b})$. This result is consistent with the findings of Rao et al. ${ }^{5}$. In addition, the Boyden chamber assay using the xCELLigence system showed that the knockdown of PLCE1 resulted in a decreased migratory phenotype in cultured podocytes (Fig. 4c).

As Rho GTPases play an important role in organizing the actin cytoskeleton, thereby affecting the cell morphology ${ }^{21}$, we examined podocyte morphology in the background of the PLCE1 knockout (KO) (Supplementary 


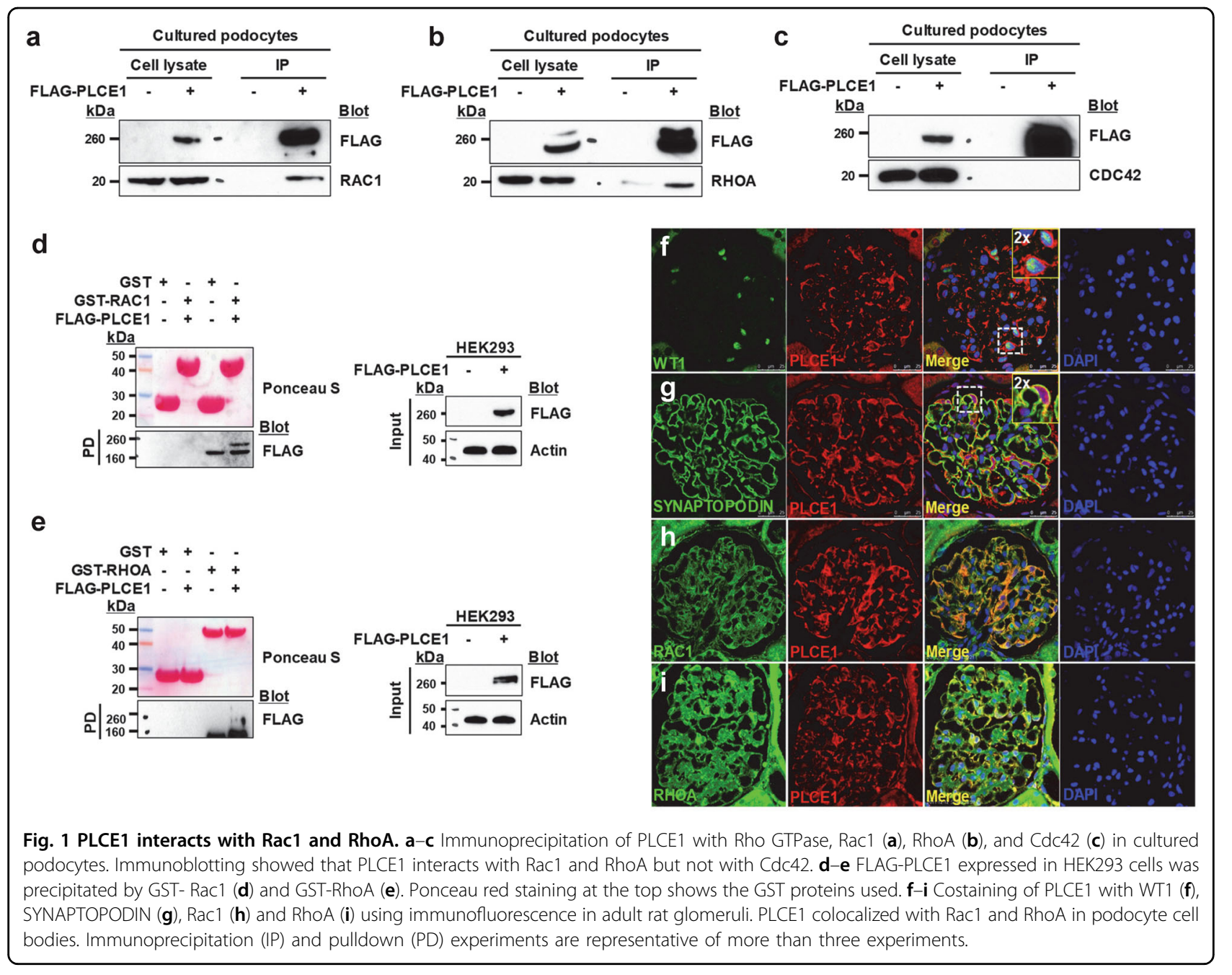

Fig. 1). PLCE1 KO resulted in the loss of terminal arborizations in undifferentiated podocytes (Fig. 4d). Furthermore, differentiated PLCE1 KO podocytes displayed increased stress fibers, reflecting damage to the podocyte architecture (Fig. 4e) $)^{22}$.

Taken together, the loss of PLCE1 resulted in decreased active Rac1 and Cdc42, thereby leading to a reduced migratory phenotype in cultured podocytes.

\section{PLCE1 interacts with NCK2 in podocytes}

NCK proteins, NCK1 and NCK2, are ubiquitously expressed adaptors that contain three Src homology 3 (SH3) domains and an $\mathrm{SH} 2$ domain; a podocyte-specific deletion of both genes leads to $\mathrm{FSGS}^{23}$. Coimmunoprecipitation was performed to investigate the interaction between PLCE1 and NCKs; this was based on the prediction of a web-based protein-protein interaction database, PrePPI (https://bhapp. c2b2.columbia.edu/PrePPI/), which showed that PLCE1 can interact with NCKs. The results showed that PLCE1 interacted with NCK2 but not with NCK1 (Fig. 5a, b).
NCKs relay signals from receptors at the plasma membrane to the actin cytoskeleton by simulating $\mathrm{N}$ WASP-Arp2/3-induced actin nucleation ${ }^{24}$ or by activating Pak $1^{25}$. As NCK2 interacts with PLCE1, the effect of NCK2 on podocyte migration was examined. Therefore, NCK2 was knocked out in cultured podocytes using the CRISPR/Cas9 system (Supplementary Fig. 2). NCK2 knockout (KO) podocytes exhibited decreased migration in a manner similar to podocytes with PLCE1 knockdown (Fig. 5c). These results suggest that PLCE1 cooperates with NCK2 to regulate the actin cytoskeleton in podocytes.

\section{PLCE1 regulates the activity of mitogen-activated protein kinases in podocytes}

PLCE1 transduces mitogenic signals through its activity as a RasGEF to stimulate the Ras and mitogen-activated protein kinase (MAPK) signaling pathway ${ }^{11,26}$. To investigate whether PLCE1 plays a similar function in podocytes, we investigated the Ras/MAPK signaling pathway. Upon 


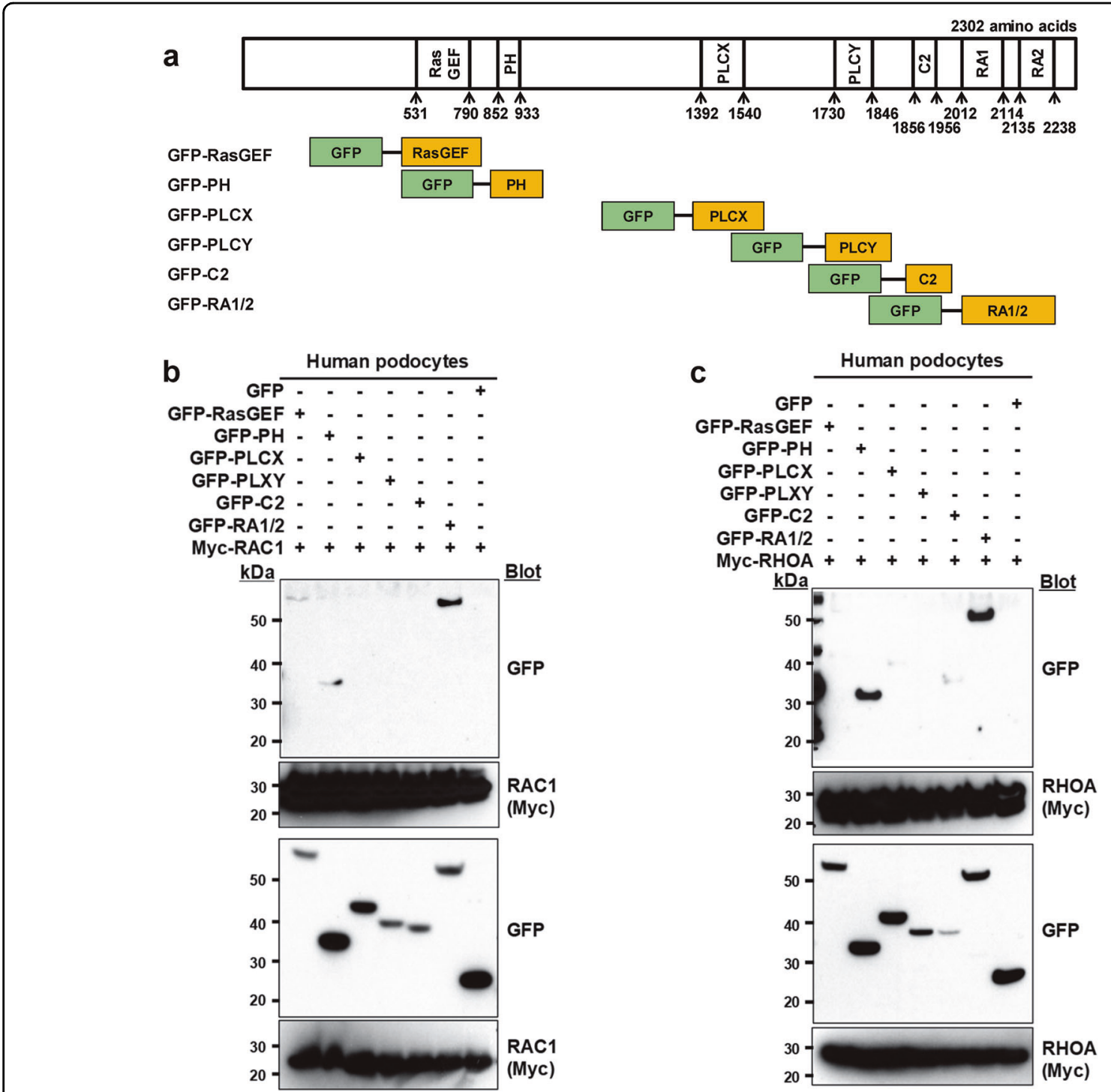

Fig. 2 The PH and RA1/2 domains of PLCE1 are required for the interaction with Rac1 and RhoA. a Schematic representation of the PLCE1 domains. PLCE1 contains a RasGEF domain, a pleckstrin homology $(\mathrm{PH})$ domain, two regions of sequence among PLCS $(\mathrm{X}$ and $\mathrm{Y})$, a C2 domain, and two Ras-binding (RA) domains. The numbers represent the amino acid positions. The fragments of PLCE1 were fused to GFP at their N-terminus. $\mathbf{b}, \mathbf{c}$ Interactions of PLCE1 domains with Rho GTPase Rac1 and RhoA. GFP-tagged PLCE1 fragments and Myc-tagged Rac1 (b) and RhoA (c) were transfected into podocytes, and immunoprecipitation was performed using anti-Myc agarose beads. Notably, both RAC1 and RhoA bind to PLCE1 through the $\mathrm{PH}$ and $\mathrm{RA} 1 / 2$ domains.

stimulation of the control podocytes with epidermal growth factor (EGF), the levels of phosphorylated extracellularsignal-regulated kinase (ERK) and c-Jun $\mathrm{N}$-terminal kinase (JNK) were increased after $45 \mathrm{~min}$, but they returned to basal levels after $120 \mathrm{~min}$ (Fig. 6a). However, the EGFinduced phosphorylation of ERK and JNK was significantly reduced in PLCE1 KO podocytes (Fig. 6a). In addition, PLCE1 KO podocytes exhibited lower proliferation than the control podocytes (Fig. 6b). However, NCK2 KO podocytes showed proliferation similar to that of control podocytes
(Fig. 6c), indicating that NCK2 is not involved in the proliferation of podocytes.

The effect of PLCE1 KO on podocyte differentiation was examined as MAPKs regulate differentiation ${ }^{27}$. When cultured at $37^{\circ} \mathrm{C}$ for 14 days, the levels of podocyte markers, such as NEPH1, NPHS1, WT1, and SYNPO, were significantly reduced in the PLCE1 KO podocytes compared to those in control podocytes, whereas no difference was observed between the NCK2 $\mathrm{KO}$ and control podocytes with respect to differentiation (Fig. 6d). 

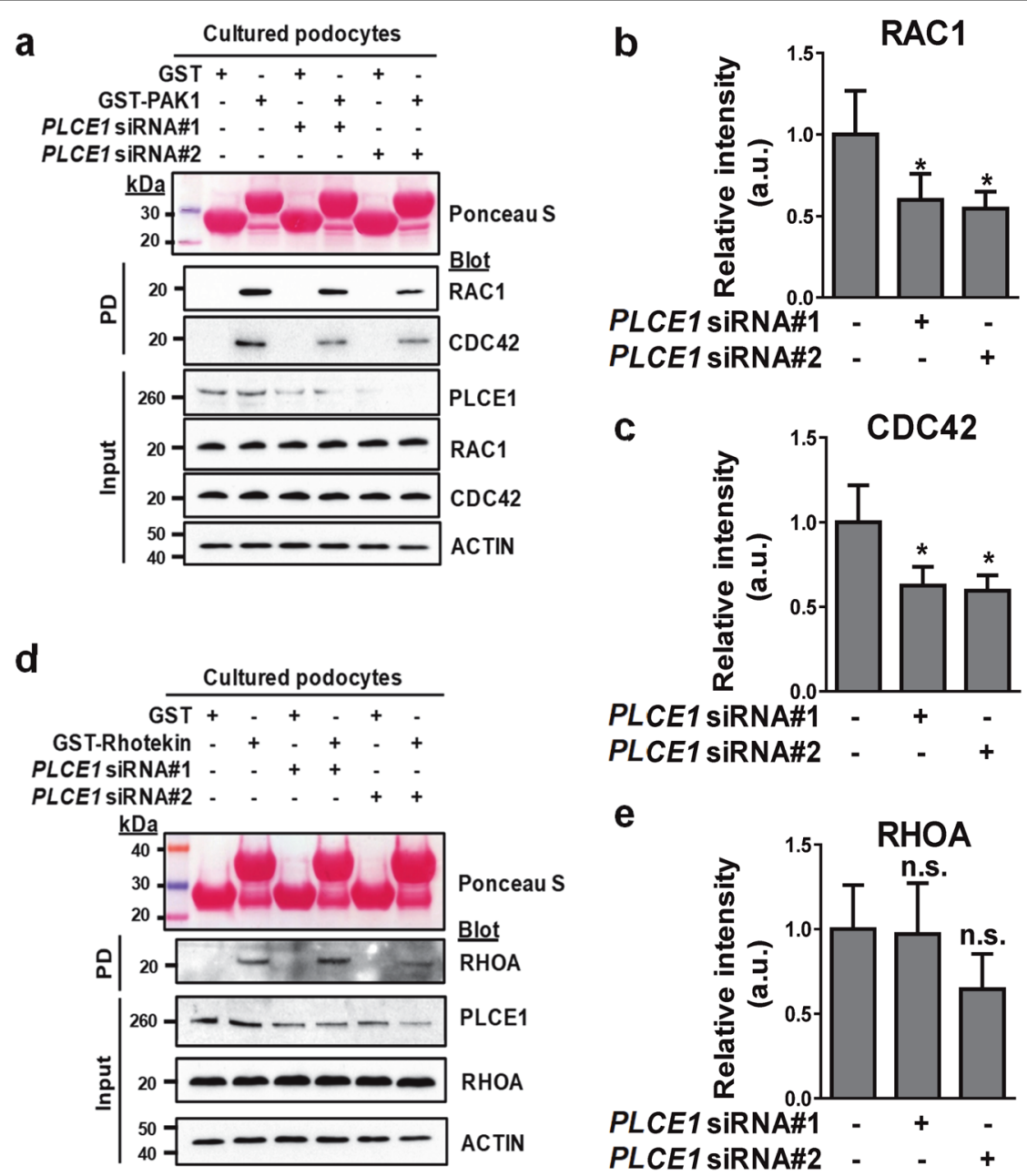

Fig. 3 The effects of the PLCE1 knockdown on Rho GTPase activity. a Active GTP-bound Rac1 and Cdc42 precipitated from podocytes transfected with PLCE1 siRNA using a GST-PAK1 (CRIB domain) pulldown assay. Ponceau red staining at the top shows the GST proteins used. Compared with control podocytes, podocytes transfected with PLCE1 siRNA exhibited a significant decrease in GTP-bound Rac1 and Cdc42 levels. b, c Quantification of Rac1 (b) and Cdc42 (c) in PLCE1 knockdown cells compared to that in control cells. d Active GTP-bound RhoA precipitated from podocytes transfected with PLCE1 siRNA using a GST-rhotekin (RBD domain) pulldown assay. Cells transfected with or without PLCE1 siRNA exhibited no significant differences in GTP-bound RhoA. e Quantification of RhoA in PLCE1 knockdown cells compared with that in control cells. Error bars indicate the standard deviations for more than three independent experiments. ${ }^{*} P<0.05$; n.s. not significant.

Taken together, PLCE1 is involved in mediating the proliferation and differentiation of podocytes by regulating MAPKs independently of NCK2.

\section{Discussion}

In this study, it was found that PLCE1 is involved in the regulation of Rho GTPases and MAPKs in podocytes (Fig. 7). It was also found that PLCE1 interacts with NCK2 but not with NCK1. PLCE1 KO podocytes exhibit a decrease in active GTP-bound Rac1 and Cdc42 and decreased migration. In addition, PLCE1 KO podocytes show a decrease in ERK phosphorylation and proliferation upon EGF stimulation and a decrease in the expression of podocyte markers upon differentiation.
PLCE1 is a multifunctional enzyme exhibiting phospholipase as well as RasGEF activities ${ }^{26}$. In podocytes, PLCE1 can be activated by the $G_{\alpha}$ protein upon the stimulation of the angiotensin II receptor by angiotensin II. Then, through its phospholipase activity, PLCE1 hydrolyzes phosphatidylinositol 4,5-bisphosphate, thereby generating DAG and $\mathrm{IP}_{3}$. DAG activates TRPC6, and IP3 activates a calcium channel located in the endoplasmic reticulum, thereby contributing to the increase in the intracellular $\mathrm{Ca}^{2+}$ concentration. In podocytes, PLC $\beta$ and PLCE1 play a redundant role in angiotensin II-induced DAG production, and angiotensin II-induced TRPC6 currents are normally generated in $P l c e 1^{-1-}$ podocytes ${ }^{15}$. 


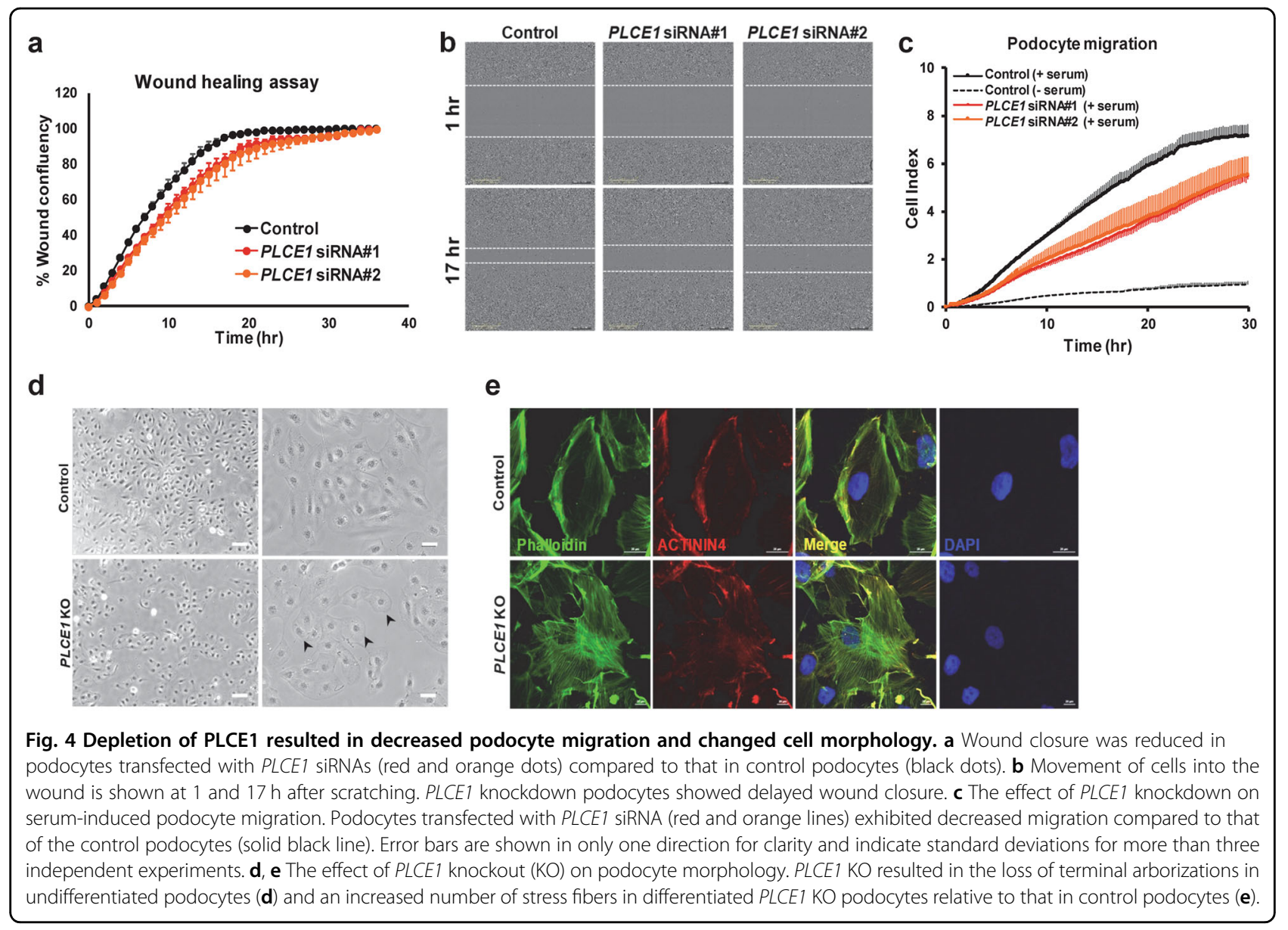

a

FLAG-PLCE1 + -+
Myc-NCK1 - + +

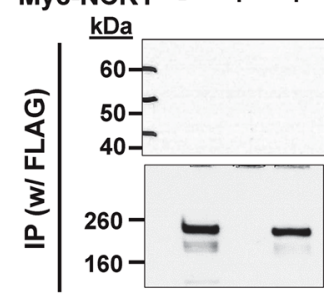

Blot

NCK1

(Myc)

PLCE1

(FLAG)

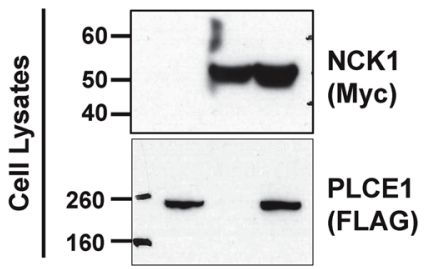

\section{b}

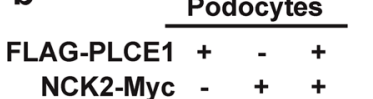

可।

$60-$
$50-$
$40-$

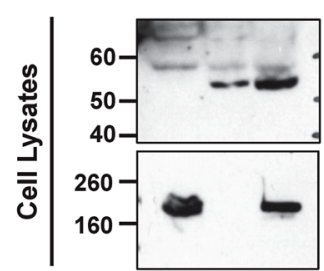

Blot

NCK2

(Myc)

PLCE1

(FLAG)

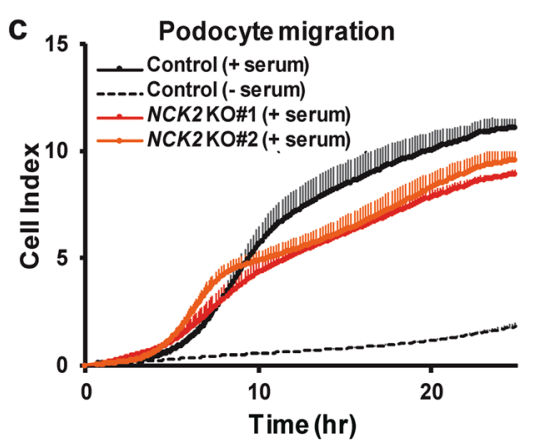

NCK2

(Myc)

PLCE1

(FLAG)

Fig. 5 NCK2 interacts with PLCE1 and affects the migration in podocytes. $\mathbf{a}$, $\mathbf{b}$ Interaction of PLCE1 and NCKs. The plasmids containing FLAGtagged PLCE1 and Myc-tagged NCKs were transfected into podocytes, and cell lysates were immunoprecipitated using anti-FLAG agarose beads. Notably, PLCE1 interacted with NCK2 (b) but not with NCK1 (a). The immunoblots are representative of more than three experiments. IP, immunoprecipitation. c The effect of NCK2 knockout (KO) on podocyte migration. NCK2-depleted podocytes exhibited decreased migration (red and orange lines) compared with that of the control podocytes (solid black line). Error bars are shown in only one direction for clarity and indicate standard deviations for more than three independent experiments. 


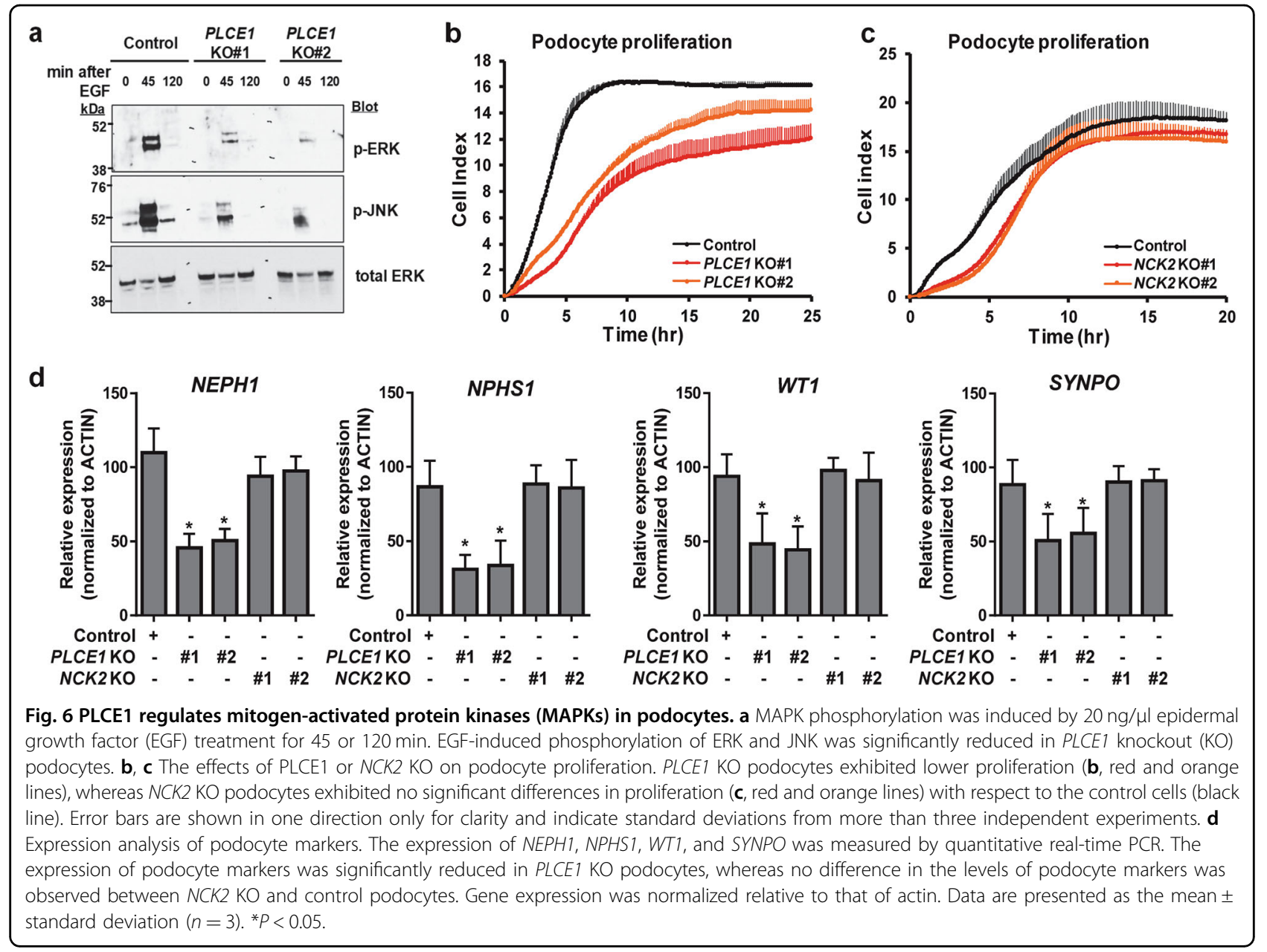

Indeed, PLC $\beta$ isoforms are essential for angiotensin IImediated activation of TRPC6 in podocytes ${ }^{28}$. Taken together, the loss of phospholipase activity is not sufficient to explain the pathogenesis of nephrotic syndrome resulting from PLCE1 mutations.

Rho GTPases are key players in the regulation of actin dynamics and cell migration ${ }^{21}$. Both increased and decreased Rho GTPase signaling interfere with podocyte mobility, thus causing proteinuria in humans and animal models ${ }^{29}$. RhoA directly activates the phospholipase activity of PLCE $1{ }^{12}$, but the active state of RhoA was not affected in PLCE1 knockdown podocytes. However, it was found that the levels of GTP-bound Rac1 and Cdc42 were decreased by the PLCE1 knockdown, which also led to a reduction in the migratory phenotype of podocytes. The podocyte-specific $C d c 42 \mathrm{KO}$ mouse model exhibits severe proteinuria and early-onset FSGS, whereas the podocyte-specific Rac1 KO mice are normal but are more susceptible to chronic hypertensive damage $^{17}$. Therefore, decreased levels of active Cdc42 and Rac1 may have roles in PLCE1 mutation-induced disease pathogenesis.
PLCE1 mutations are histologically associated with $\mathrm{DMS}^{8,9}$, which is known to be characterized by a diffuse increased mesangial matrix and immature glomeruli ${ }^{30}$. In addition, podocytes are hypertrophic and have a cuboidal (immature) appearance ${ }^{30}$. In this study, PLCE1 KO podocytes exhibited reduced MAPK activation and cell proliferation upon EGF stimulation. In addition, PLCE1 KO podocytes expressed low levels of podocyte markers upon differentiation, indicating that they remain immature. These characteristics of PLCE1 KO podocytes could explain the DMS observed upon analyzing the renal histology in individuals with PLCE1 mutations.

NCK1 and NCK2 exhibited redundancy in podocytes, as the simultaneous deletion of both genes, but not that of a single gene, resulted in podocytopathy ${ }^{23}$. However, they also have distinct functions. For example, NCK1, but not $\mathrm{NCK2}$, is ubiquitinated by c-Cbl, which competes with synaptopodin for binding to NCK1 and protects NCK1 from proteasomal degradation. Then, NCK1 induces the formation of stress fibers in cooperation with $\mathrm{RhoA}^{31}$. In this study, it was found that PLCE1 interacts with NCK2 but not with NCK1. NCK2 KO resulted in the reduced 


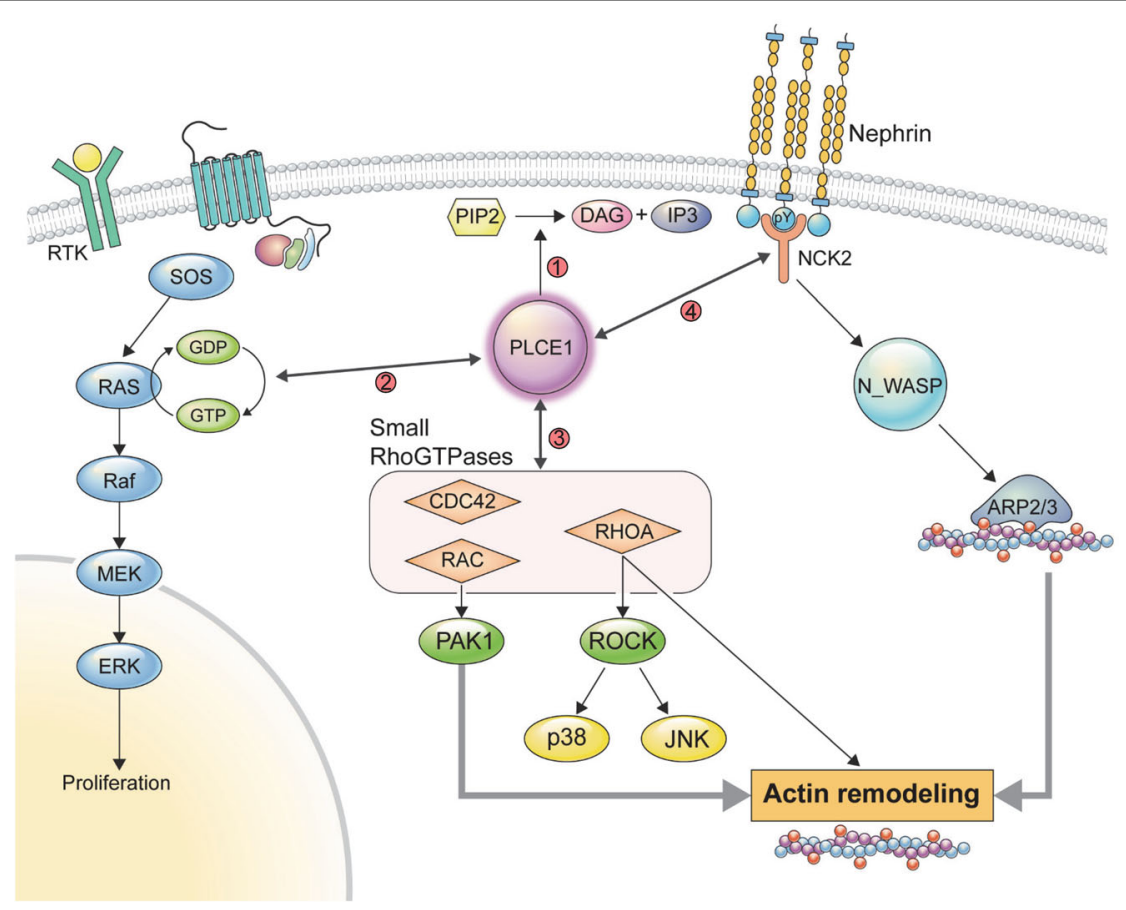

Fig. 7 Functions of PLCE1 in podocytes. PLCE1 plays multiple roles in podocytes: (1) PLCE1 acts as a phospholipase and degrades phosphatidylinositol 4,5-bisphosphate (PIP 2 ) into diacylglycerol (DAG) and inositol triphosphate (IP $\mathrm{P}_{3}$ ). (2) PLCE1 can be activated by a G-protein and transduces mitogenic signals through its RasGEF activity to stimulate the Ras and MAPK pathways. (3) PLCE1 regulates the activity of Rho GTPases, which are the key regulators of actin dynamics and cell migration. Upon loss of PLCE1, the levels of active Rac1 and Cdc42 were reduced. (4) PLCE1 interacts with NCK2, which plays a pivotal role in stimulating N-WASP-Arp2/3-induced actin nucleation.

migration of podocytes in a manner similar to that observed upon PLCE1 knockdown. As the levels of active Rac1 and Cdc42 were reduced upon PLCE1 knockdown, it will be interesting to examine whether NCK2 regulates Rac1 and Cdc42 along with PLCE1 in podocytes.

We revealed that PLCE1 plays multiple roles in podocytes. Mutational analysis of PLCE1 revealed that mutations are distributed uniformly throughout $P L C E 1^{32}$. Some frameshift mutations at the C-terminus of PLCE1 affect one or both of the RA domains, but the protein retains intact phospholipase and RasGEF activities. It was found that the RA domain is necessary for interacting with Rac1 and RhoA, suggesting that these mutations may be pathogenic as they dysregulate Rho GTPase signaling.

\section{Acknowledgements}

We thank the Yonsei Advanced Imaging Center along with Carl Zeiss Microscopy. H.Y.G. was supported by the Research Program of the National Research Foundation of Korea (NRF) funded by the Korean government (MSIT 2018R1A5A2025079). We thank MID (Medical Illustration \& Design) for providing support for the medical illustrations.

\section{Conflict of interest}

The authors declare that they have no conflict of interest.

\section{Publisher's note}

Springer Nature remains neutral with regard to jurisdictional claims in published maps and institutional affiliations.
Supplementary information accompanies this paper at https://doi.org/ 10.1038/s12276-020-0410-4.

Received: 3 October 2019 Revised: 7 February 2020 Accepted: 12 February 2020.

Published online: 1 April 2020

\section{References}

1. Tryggvason, K., Patrakka, J. \& Wartiovaara, J. Hereditary proteinuria syndromes and mechanisms of proteinuria. N. Engl. J. Med. 354, 1387-1401 (2006).

2. Somlo, S. \& Mundel, P. Getting a foothold in nephrotic syndrome. Nat. Genet. 24, 333-335 (2000).

3. Hildebrandt, F. Genetic kidney diseases. Lancet (Lond., Engl.) 375, 1287-1295 (2010).

4. Sadowski, C. E. et al. A single-gene cause in $29.5 \%$ of cases of steroid-resistant nephrotic syndrome. J. Am. Soc. Nephrol. 26, 1279-1289 (2015).

5. Rao, J. et al. Advillin acts upstream of phospholipase $C 1$ in steroid-resistant nephrotic syndrome. J. Clin. Investig. 127, 4257-4269 (2017).

6. Somlo, S. \& Mundel, P. Getting a foothold in nephrotic syndrome. Nat. Genet. 24, 333 (2000).

7. Kriz, W. Podocyte is the major culprit accounting for the progression of chronic renal disease. Microsc Res Tech. 57, 189-195 (2002).

8. Hinkes, B. et al. Positional cloning uncovers mutations in PLCE1 responsible for a nephrotic syndrome variant that may be reversible. Nat. Genet. $\mathbf{3 8}$, 1397-1405 (2006).

9. Gbadegesin, R. et al. Mutations in PLCE1 are a major cause of isolated diffuse mesangial sclerosis (IDMS). Nephrol. Dial. Transplant. 23, 1291-1297 (2008).

10. Smrcka, A. V., Brown, J. H. \& Holz, G. G. Role of phospholipase C $\varepsilon$ in physiological phosphoinositide signaling networks. Cell Signal 24, 1333-1343 (2012). 
11. Citro, S. et al. Phospholipase Cepsilon is a nexus for Rho and Rap-mediated G protein-coupled receptor-induced astrocyte proliferation. Proc. Natl Acad. Sci. USA 104, 15543-15548 (2007).

12. Wing, M. R., Snyder, J. T., Sondek, J. \& Harden, T. K. Direct activation of phospholipase C-epsilon by Rho. J. Biol. Chem. 278, 41253-41258 (2003).

13. Winn, M. P. et al. A mutation in the TRPC6 cation channel causes familial focal segmental glomerulosclerosis. Science 308, 1801-1804 (2005).

14. Reiser, J. et al. TRPC6 is a glomerular slit diaphragm-associated channel required for normal renal function. Nat. Genet. 37, 739-744 (2005).

15. Kalwa, $H$. et al. Phospholipase C Epsilon (PLC $(\varepsilon)$ Induced TRPC6 Activation: A Common but Redundant Mechanism in Primary Podocytes. J. Cell. Physiol. 230, 1389-1399 (2015).

16. Gee, H. Y. et al. ARHGDIA mutations cause nephrotic syndrome via defective RHO GTPase signaling. J. Clin. Investig. 123, 3243-3253 (2013).

17. Blattner, S. M. et al. Divergent functions of the Rho GTPases Rac1 and Cdc42 in podocyte injury. Kidney Int. 84, 920-930 (2013).

18. Scott, R. P. et al. Podocyte-specific loss of Cdc42 leads to congenital nephropathy. J. Am. Soc. Nephrol. 23, 1149-1154 (2012).

19. Zhu, L., Jiang, R., Aoudjit, L., Jones, N. \& Takano, T. Activation of RhoA in podocytes induces focal segmental glomerulosclerosis. J. Am. Soc. Nephrol. 22, 1621-1630 (2011).

20. Gee, H. Y. et al. KANK deficiency leads to podocyte dysfunction and nephrotic syndrome. J. Clin. Investig. 125, 2375-2384 (2015).

21. Garcia-Mata, R., Boulter, E. \& Burridge, K. The 'invisible hand': regulation of RHO GTPases by RHOGDIs. Nat. Rev. Mol. Cell Biol. 12, 493-504 (2011).

22. Kistler, A. D., Altintas, M. M. \& Reiser, J. Podocyte GTPases regulate kidney filter dynamics. Kidney Int. 81, 1053-1055 (2012).
23. Jones, N. et al. Nck proteins maintain the adult glomerular filtration barrier. J. Am. Soc. Nephrol. 20, 1533-1543 (2009).

24. Rohatgi, R., Nollau, P., Ho, H. Y., Kirschner, M. W. \& Mayer, B. J. Nck and phosphatidylinositol 4,5-bisphosphate synergistically activate actin polymerization through the N-WASP-Arp2/3 pathway. J. Biol. Chem. 276, 26448-26452 (2001)

25. Lu, W., Katz, S., Gupta, R. \& Mayer, B. J. Activation of Pak by membrane localization mediated by an $\mathrm{SH} 3$ domain from the adaptor protein Nck. Curr. Biol. 7, 85-94 (1997).

26. Lopez, I., Mak, E. C., Ding, J., Hamm, H. E. \& Lomasney, J. W. A novel bifunctional phospholipase $c$ that is regulated by Galpha 12 and stimulates the Ras/ mitogen-activated protein kinase pathway. J. Biol. Chem. 276, 2758-2765 (2001).

27. Zhang, W. \& Liu, H. T. MAPK signal pathways in the regulation of cell proliferation in mammalian cells. Cell Res. 12, 9-18 (2002).

28. Gudermann, T. A new TRP to kidney disease. Nat. Genet. 37, 663-664 (2005).

29. Lal, M. A. \& Tryggvason, K. Knocking out podocyte rho GTPases: and the winner is. J. Am. Soc. Nephrol. 23, 1128-1129 (2012).

30. Barisoni, L., Schnaper, H. W. \& Kopp, J. B. Advances in the biology and genetics of the podocytopathies: implications for diagnosis and therapy. Arch. Pathol. Lab. Med. 133, 201-216 (2009).

31. Buvall, L. et al. Proteasomal degradation of Nck1 but not Nck2 regulates RhoA activation and actin dynamics. Nat. Commun. 4, 2863 (2013).

32. Boyer, $\mathrm{O}$. et al. Mutational analysis of the PLCE1 gene in steroid resistant nephrotic syndrome. J. Med. Genet. 47, 445-452 (2010). 